\title{
Lessons learned from the coronavirus disease 2019 (Covid-19) outbreak in a monographic center for spinal cord injury
}

\author{
Elisa López-Dolado $\mathbb{B}^{1} \cdot$ Angel Gil-Agudo $^{1}$
}

Received: 12 April 2020 / Accepted: 14 April 2020 / Published online: 28 April 2020

(c) International Spinal Cord Society 2020

For just over three months, our whole world has just been turned upside down. In late December 2019, an epidemic of severe acute respiratory syndrome coronavirus 2 (SARSCoV-2) started in Wuhan [1] and spread rapidly throughout the world. This pandemic reached us not silently but abruptly, even brutally. The World Health Organization (WHO) declared the 2019 coronavirus disease (Covid-19) a pandemic on March 11, 2020 and as of April 2, 2020, $1.002,159$ cases were confirmed worldwide with 51,485 deaths [2]. The new coronavirus, responsible for the severe acute respiratory syndrome (SARS- CoV-2), has brought higher levels of illness, death, and fear throughout the planet greater than the current generation has known until now [2]. As Spaniards we are a good example of how adversity brings out the true measure of a society. Since the first Covid-19 confirmed case in La Gomera (Canary Islands), our clinical role in the pandemic has been smoothly but constantly changing [3].

In this context, what about people with spinal cord injury (SCI)? SCI induces numerous chronic disorders that put these individuals at a high risk of severe Covid-19 prognosis. Specifically, the SCI population presents higher rates of hypertension, SCI-induced immunosuppression and, if the metameric level is T8 or above, respiratory failure with continuous or episodic hypoxemia due to respiratory muscle weakness. Sympathetic denervation following SCI compromises body temperature regulation, as a result of dysautonomia, which not only complicates the adequate early

Supplementary information The online version of this article (https:// doi.org/10.1038/s41393-020-0473-z) contains supplementary material, which is available to authorized users.

Angel Gil-Agudo

amgila@sescam.jccm.es

1 Department of Rehabilitation, Hospital Nacional de Parapléjicos of Toledo, Toledo, Spain diagnosis of Covid-10 so that patients are at risk of a poor prognosis, but also makes it difficult to control infection transmission to other patients and/or healthcare staff.

With so many potential patients on the horizon and so many brilliant clinicians and colleagues dealing with the consequences of Covid-19, how could we be of use for people/patients with SCI and to the medical community?

The first Covid-19 case at the Hospital Nacional de Parapléjicos (HNP) of Toledo was confirmed on March 17, 2020 , following close contact with an infected relative. As the HNP is the national SCI monographic hospital in Spain with 212 beds, classified as a long-term healthcare facility, our institution immediately launched a contingency plan with the following objectives: (1) avoid the spread of Covid19 among non-infected people/patients with SCI and healthcare staff; (2) treat adequately SCI Covid-19 confirmed cases; (3) design, create and apply the infrastructure to perform face-to-face and remote ongoing rehabilitation treatment using telemedicine support; (4) continue admission of new patients with acute SCI using enhanced hygiene protection conditions; and (5) make available all the clinical resources at its disposal to the Regional Public Health Service to help manage patients without SCI but with confirmed Covid-19 infection, in need of hospital admission.

In order to avoid the spread of infection, one of our first goals was to decrease the traffic of people through the hallways and corridors: the physiotherapy and occupational therapy treatment rooms are temporarily closed, carrying out all the necessary rehabilitation treatment inside the patients' rooms; those patients whose SCI is stable enough are sent home to quarantine, with periodic telephone follow-up by the hospital staff; and all visits to the inpatient area are forbidden. The seven people/patients with SCI diagnosed with a confirmatory RT-PCR test for Covid-19 infection were transferred to isolation zones in an inpatient area with adequate space, and cared for by healthcare personnel wearing personal protection equipment. This area is directly under the supervision of our Internal Medicine staff physicians. Hospitalized 
people/patients with SCI without Covid-19 continue their rehabilitation program in their rooms. To facilitate the continuity of home treatment in patients who undergoing quarantine, new materials suitable for telemedicine have been designed and created, using various types of support: a series of videos newly recorded with physiotherapy programs, occupational therapy guidelines, with the doctors' recommendations aimed at preventing pressure ulcers, controlling spasticity or pain, and/or reminding patients about appropriate prevention measures against Covid-19. All these new materials have been disseminated by social media including Twitter (@HNParaplejicos). Furthermore, an English translation of the HNP physiotherapy respiratory rehabilitation protocol for patients with SCI and Covid-19 has been translated and sent to the Covid-19 Resources page, where other useful guidelines and factsheets can be found (https://www. iscos.org.uk//covid-19-resources-). New acute people/patients with SCI are admitted for seven days to a different inpatient unit, in single-use rooms. If symptoms suggestive of Covid19 infection develop (fever, cough, dyspnea), or suspicious analytical parameters are detected, diagnostic confirmation by RT-PCR is requested. If Covid-19 infection is confirmed, the patient is sent directly to the SCI Covid-19 inpatient area (named above). If the confirmatory test is negative or the seven-day observation period is completed without symptoms, the patient is transferred to a conventional room to continue their rehabilitation programs. Since these new measures have been implemented, we have identified seven people/patients with SCI with confirmed Covid-19, but no new cases have been found to date (April 10, 2020).

As might be expected, we cannot forget the critical situation our National Health System is going through at present, so we have made available to the Regional Health Department a total of 90 inpatient rooms with an Intensive Care area able to attend ten more critically ill people with Covid-19 infection from the general population in need of hospital admission. In order to avoid infection spreading as much as possible, two sealed circuits have been created with completely independent spaces separating the patients from the people/patients with SCI.

We all know from first-hand experience how many complications affect people with SCI and how vulnerable they can be. However, in recent years our ability to take care of people with SCI has improved so much that we have dared to dream about the possibilities of novel therapies that could dramatically improve essential function and quality of life. But now, because of the Covid-19 pandemic, the immediate heath priorities have drastically changed and we face, as with geriatricians, the fact that the people/patients with SCI belong to the population group with a high risk of suffering severe Covid-19 complications and death. We had feared that because of SCI-induced systemic immunosuppression [4-6], autoimmunity [7] and respiratory failure, patients with SCI who are diagnosed with Covid-19 infection would have to face a poor evolution and harsh prognosis. But our findings do not agree with these pessimistic expectations. Despite the presentation of the Covid-19 clinical picture (phenotype) the current patient case series [8], consisting mainly of males aged over 60 years who are tracheostomized due to their subacute and complete cervical SCI, have shown a lower incidence of symptoms at the onset of infection with more benign outcomes compared to the general population.

Of course, our data represent the clinical behavior of a limited number of patients. But what if the blunted SCI immune response profile actually leads to a milder clinical presentation? Understanding the underlining immune response in people with SCI could help us to better understand the Covid-19 response in the general population. By doing what we do best in the hospital - keeping a close watch on patients with SCI - we are also playing our part to combat the present pandemic by also helping not to forget about the search for how the patient's response to infection may also lead to an improvement in their general complications as a consequence of SCI: the intimate hope of all of people/patients with SCI patients.

It is very important to know the evolution of the disease following Covid-19 infection in people with SCI during this pandemic period and the preventive management procedures carried out in spinal centers where these patients are treated. We should not forget that we still do not have a universally accepted treatment or preventive vaccine. Everything suggests that in the not too distant future there will be new epidemic outbreaks. We feel that it is important to document our current clinical procedures (see Supplementary File) to the wider community, because the knowledge acquired during the current episode may need to be applied again soon, so that better clinical and resource management measures can be applied early to avoid possible ineffective actions taken in the near future.

Acknowledgements Critical and English revision by Julian Taylor $\mathrm{PhD}$, Hospital Nacional de Parapléjicos of Toledo (Spain).

\section{Compliance with ethical standards}

Conflict of interest The authors declare that they have no conflict of interest.

Publisher's note Springer Nature remains neutral with regard to jurisdictional claims in published maps and institutional affiliations.

\section{References}

1. Li Q, Guan X, Wu P, Wang X, Zhou L, Tong Y, et al. Early transmission dynamics in Wuhan, China, of novel coronavirusinfected pneumonia. N. Engl J Med. 2020;382:1199-207. 
2. Coronavirus COVID-19 Global Cases by the Center for Systems Science and Engineering (CSSE) at Johns Hopkins University (JHU). JHU COVID-19 Resource Center. Johns Hopkins Coronavirus Resource Center. https://www.arcgis.com/apps/opsdashboard/index. html\#/bda7594740fd40299423467b48e9ecf6. Accessed 2 April 2020.

3. Protocolo de actuación frente a casos de infección por el Nuevo Coronavirus (SARS-CoV-2). Ministerio de Sanidad. Gobierno de España. https://www.mscbs.gob.es/profesionales/saludPublica/cca yes/alertasActual/nCovChina/documentos/Procedimiento_COVID_ 19.pdf. Accessed 4 April 2020.

4. Legido-Quigley H, Mateos-García JT, Regulez Campos V, Gea-Sánchez M, Muntaner C, McKee M. The resilience of the Spanish health system against the COVID-19 pandemic. Lancet Public Health 2020. Published Online March 18, 2020. https://doi. org/10.1016/S2468-2667(20)30060-8.
5. Riegger T, Conrad S, Liu K, Schluesener HJ, Adibzahdeh M, Schwab JM. Spinal cord injury-induced immune depression syndrome (SCI-IDS). Eur J Neurosci. 2007;25:1743-7.

6. Prüss H, Tedeschi A, Thiriot A, Lynch L, Loughhead SM, Stutte S, et al. Spinal cord injury-induced immunodeficiency is mediated by a sympathetic-neuroendocrine adrenal reflex. Nat Neurosci 2017;20: 1549-59.

7. Arévalo-Martin A, Grassner L, Garcia-Ovejero D, Paniagua-Torija B, Barroso-Garcia G, Arandilla AG, et al. Elevated autoantibodies in subacute human spinal cord injury are naturally occurring antibodies. Front Immunol 2018;9:2365.

8. Rodríguez-Cola M, Jiménez Velasco I, Gutiérrez Henares F, López Dolado E, Gambarrutta Malfatti C, Gil-Agudo. A Clinical features of coronavirus disease 2019 (COVID-19) in a cohort of spinal cord injured patients. Spinal Cord Ser Cases. Under review, 2020. 